\title{
Ulnar Nerve Injury Caused by the Incomplete Insertion of a Screw Head after Internal Fixation with Dual Locking Plates in AO/OTA Type C2 Distal Humerus Fractures
}

\author{
Jae-Hyuk Shin, Whan-Jin Kwon, Yoon-Suk Hyun \\ Department of Orthopaedic Surgery, Hallym University Kangdong Sacred Heart Hospital, Seoul, Korea
}

\begin{abstract}
After dual plating with a locking compression plate for comminuted intraarticular fractures of the distal humerus, the incidence of ulnar nerve injury after surgery has been reported to be up to $38 \%$. This can be reduced by an anterior transposition of the ulnar nerve but some surgeons believe that extensive handling of the nerve with transposition can increase the risk of an ulnar nerve dysfunction. This paper reports ulnar nerve injuries caused by the incomplete insertion of a screw head in dual plating without an anterior ulnar nerve transposition for AO/OTA type C2 distal humerus fractures. When an anatomical locking plate is applied to a distal humeral fracture, locking screws around the ulnar nerve should be inserted fully without protrusion of the screw because an incompletely inserted screw can cause irritation or injury to the ulnar nerve because the screw head in the locking system usually has a slightly sharp edge because screw head has threads. If the change in insertion angle and resulting protruded head of the screw are unavoidable for firm fixation of fracture, the anterior transposition of the ulnar nerve is recommended over a soft tissue shield.
\end{abstract}

(Clin Shoulder Elbow 2017;20(4):236-239)

Key Words: AO type C; Distal humerus fractures; Ulnar nerve; Internal fixation

Dual plating with a locking compression plate for comminuted intraarticular fractures of the distal humerus is popular method. ${ }^{1-4)}$ The incidence of ulnar nerve injury after surgery has been reported to range from $10 \%$ to $38 \%$,6) and is reduced by the anterior transposition of ulnar nerve. ${ }^{6}$ This prevalence of injury is multifactorial and iatrogenic ulnar neuropathy might be the result of several causes, including manipulation during splinting, the proximity of the nerve to the injury zone, intraoperative manipulation, entrapment in scar tissue, or hardware irritation. ${ }^{7,8)}$ Among them, this paper reports ulnar nerve neuropathy irritated by an incompletely inserted screw head in dual plating with a locking compression system.

\section{Case Report}

A thirty nine year-old female was transferred with a type C2
(AO/OTA classification) distal humerus fracture and a preoperative computed tomography $(\mathrm{CT})$ scan. The patient did not have any signs and symptoms of nerve injury below the elbow.

After 2 days, fracture was fixed by dual plating with a locking compression plate (Zimmer Biomet, Warsaw, IN, USA) through the posterior approach without an olecranon osteotomy. First, the ulnar nerve was identified and the surrounding soft tissues were released from the arcade of Struthers, which is a thin aponeurotic band extending from the medial head of the triceps to the medial intermuscular septum and lies approximately $8 \mathrm{~cm}$ proximal to the medial epicondyle, to the area just proximal of the nerve branch for the flexor carpi ulnaris with the aim of preserving the nerve vascularization. The ulnar nerve was then isolated and tagged with fine rubber tubes to minimize the level of iatrogenic damage during fracture fixation. The loupe magnification was not used during the isolation procedure of

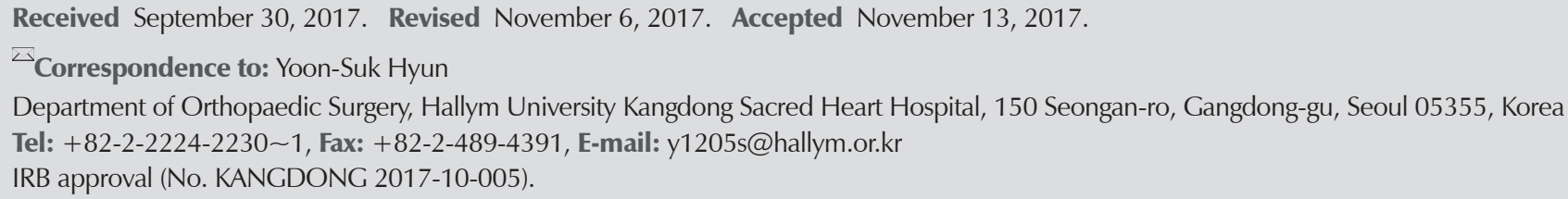


the ulnar nerve because the surgeon was not unfamiliar with it. The anatomical plates for the distal humeral fracture used in this patient were made from stainless steel and had a relative thick profile. The plates were very hard to contour and plate did not fit the patient's bone contour. In ulnar plating, it was necessary to move the position of the plate in the posterior direction rather than additional contouring to allow the optimal fixation of the locking screws into fracture fragments. The relatively short length of the ulnar side screws due to posterior movement of the medial side plate did not appear to accomplish the firm fixation. A hinged external fixator (BK Meditech, Seoul, Korea) was also applied because firm fixation could not be confirmed even after dual plating. Before external fixation, an examination was made to identify any structural damage to the ulnar nerve and determine if the ulnar nerve had been dislocated from the original site throughout the range of elbow motion. A soft tissue shield was made with the fascia of the common flexor muscles and triceps muscle around the medial epicondyle to prevent contact between the ulnar nerve and plate instead of the anterior transposition of the ulnar nerve because the transposed ulnar nerve appeared tensed on the plate even after sufficient soft tissue release for transposition.

After closing the skin incision for plating, self-tapping Shanz screws were inserted from lateral side with medial direction, two screws into the humerus, and another two screws into the ulna for external fixation. To prevent iatrogenic injury to the radial and ulnar nerves, it was confirmed that the screw did not contact the radial nerve through the mini-open procedure and did not protrude too much on the medial cortex of the ulna with fluoroscopy. On the first day after surgery, the patient did not complain of symptoms related to nerve irritation.

Four days after surgery, the patient began to complain of a severe tingling sensation and numbness on the 4th and 5th fingers; the symptoms did not improve for another 21 days with oral medication (Prednisolone, $5 \mathrm{mg} / \mathrm{d}$ for 2 weeks) and immobilization with a long arm splint. The patient showed some progression of the ulnar nerve injury with clawing of the 4th and 5th fingers, difficulty in writing with a pencil as well as existing sensory changes.

Explorative surgery was performed for unimproved ulnar nerve symptoms and sign on 28 days after the index surgery. The soft tissue shield made in the index surgery could not been found and the ulnar nerve was in contact with the head of the second distal screw in the medial plate and this screw was not fully inserted (Fig. 1A, B). Some dark color changes with a $1 \mathrm{~mm}$ diameter were observed on the contact area of the ulnar nerve (Fig. 1A). The screw was removed and a firm soft tissue shield was made again instead of nerve transposition because the ulnar nerve still remained in the ulnar groove during passive flexion and extension of the elbow and the tensed ulnar nerve was identified again on the plate with temporary anterior transposition (Fig. 1C, D).
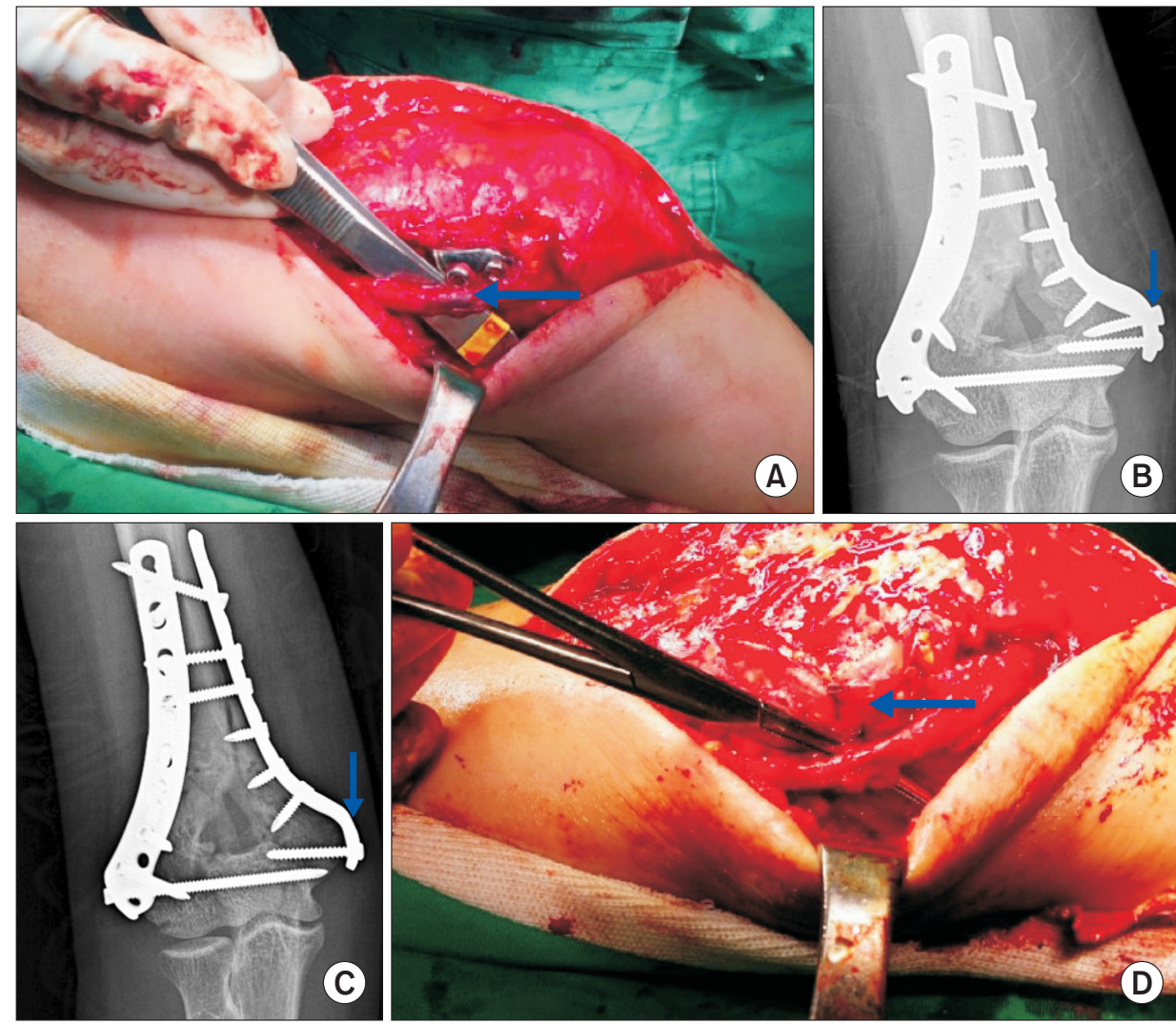

Fig. 1. (A) Soft tissue shield made in the first surgery disappeared and the ulnar nerve was in contact with the protruded head of the second distal screw in the medial plate and some dark color change (arrow) with a $1 \mathrm{~mm}$ diameter was observed on the inner surface of the ulnar nerve. This screw was not fully inserted and protruded. (B) Simple radiograph show screw head (arrow) is not fully seated. (C) Protruded screw was removed (arrow) to avoid additional irritation to the ulnar nerve. (D) Soft tissue shield (arrow) instead of nerve transposition was made again because ulnar nerve still remained in the ulnar groove during passive flexion and extension of the elbow and the tensed ulnar nerve was identified with preliminary transposition. 
The Disabilities of the Arm, Shoulder and Hand score was 80 at the visit for plate removal and 79.2 at the final visit (two and half years after the index surgery). Bony union was identified by simple radiography and a CT scan at 6 months after the index surgery. The plates were removed at one year after the index surgery. In surgery for plate removal, there were no significant anatomical changes in the nerve surface and the intact soft tissue shield made in the second explorative surgery and the ulnar nerve remained in situ after removing the plate. Electromyography (EMG) at 6 weeks and three months after the index surgery showed the severe functional disturbance of the ulnar nerve but no significant improvement was observed at the third and final EMG evaluation two and half years after the index surgery.

\section{Discussion}

Late ulnar neuropathy following open reduction and internal fixation (ORIF) of a distal humerus fracture is most common in AO type $\mathrm{C}$, followed by $\mathrm{AO}$ type $\mathrm{A}$ injuries. ${ }^{6}$

Vazquez et al. ${ }^{9)}$ examined retrospectively 69 distal humerus fractures (both $\mathrm{AO}$ type $\mathrm{A}$ and $\mathrm{C}$ ) without preoperative ulnar nerve symptoms to determine the incidence of late ulnar nerve dysfunction at a minimum of 12-month postoperatively. They reported an incidence of $10.1 \%$ immediately after surgery and $16 \%$ at the final follow-up.

Ruan et al. ${ }^{10)}$ examined 117 consecutive $\mathrm{AO}$ type $\mathrm{C}$ distal humerus fractures retrospectively and found that no patients without preoperative ulnar nerve symptoms developed late symptoms, whereas $31 \%$ of 29 patients with preoperative ulnar nerve symptoms showed late ulnar nerve symptoms.

The options for avoiding postoperative ulnar nerve symptoms include attempting to leave the nerve in situ; partially or completely releasing the nerve and moving it during surgery, then replacing it back in the cubital tunnel at the end of surgery; or completely releasing the nerve and leaving it in an anteriorly transposed position at the end of surgery.

Numerous recommendations have been made regarding when to transpose the ulnar nerve in distal humerus fracture management, even though there is no clear consensus regarding when the ulnar nerve is transposed during surgery. One investigator used the indications of preoperative palsy, possible implant irritation, or intraoperative traction. Some investigators preferred to routinely transpose the ulnar nerve in all intrarticular distal humerus fractures and appeared to favor anterior transposition of the ulnar nerve for both the prevention and treatment of neuropathy. ${ }^{3,46)}$ On the other hand, some studies reported that transposition is not protective and they did not recommend routine transposition of the ulnar nerve at the time of the ORIF of distal humerus fractures. ${ }^{7,9)}$

In the current patient, there were no preoperative ulnar nerve symptoms and the temporary transposed nerve appeared tensed on the plate. A soft tissue shield was then made between the ulnar nerve in situ and plate rather than anterior transposition.

Regarding the locking compression plating system, the screw head can be seated fully if the insertion angle is parallel to the guided angle of the screw hole (Fig. 2A, B). The screw head is not fully seated if the insertion angle is not parallel to the guided
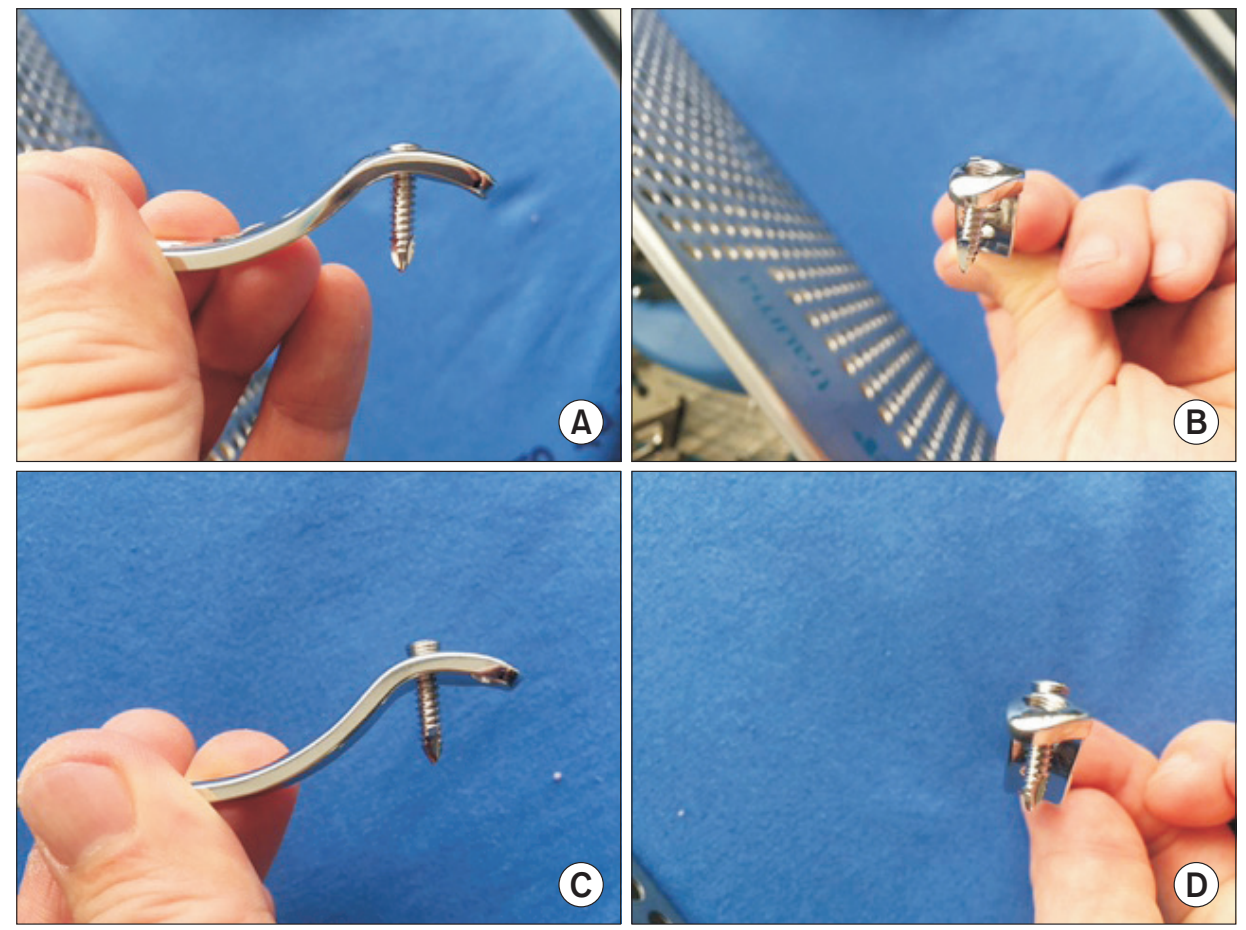

Fig. 2. (A, B) Screw head can be fully seated if the insertion angle is parallel to the guided angle of the screw hole. (C, D) Screw head was not fully seated, if the insertion angle was not parallel to the guided angle. 
angle (Fig. 2C, B). The protruded screw was inserted with a change in insertion angle because the screw did not purchase bone. The change in insertion angle for the best purchase for the bone fragment resulted in incomplete screw insertion.

The main cause of ulnar nerve palsy in the present case is believed be the direct irritation of the protruded screw head. In addition, the increased tension on the nerve in situ on the plate also could contribute to the postoperative ulnar nerve palsy as well as the protruded screw. The dark color spot on the contact surface of the ulnar nerve and the short period of the development of nerve symptoms indicated the sharp edge of the protruded screw head to be the main cause.

When applying an anatomical locking plate in distal humeral fractures, locking screws around the ulnar nerve should be fully inserted without screw protrusion because an incompletely inserted screw can cause irritation or injury to the ulnar nerve as the screw head in the locking system usually has a slightly sharp edge due to the presence of threads. If a change in the insertion angle and resulting protruded head of the screw are unavoidable, for firm fixation of a fracture, the anterior transposition of the ulnar nerve is recommended over a soft tissue shield. If a soft tissue shield between the implant and ulnar nerve is chosen instead of the anterior transposition of the nerve because the transposed nerve appears tensed on the ulnar side plate, it should not be made too firm.

\section{References}

1. Reising K, Hauschild O, Strohm PC, Suedkamp NP. Stabilisation of articular fractures of the distal humerus: early experience with a novel perpendicular plate system. Injury. 2009;40(6):611-7.
2. Sanchez-Sotelo J, Torchia ME, O'Driscoll SW. Complex distal humeral fractures: internal fixation with a principle-based parallel-plate technique. J Bone Joint Surg Am. 2007;89(5):961-9.

3. Gofton WT, Macdermid JC, Patterson SD, Faber KJ, King GJ. Functional outcome of $\mathrm{AO}$ type $\mathrm{C}$ distal humeral fractures. J Hand Surg Am. 2003;28(2):294-308.

4. Athwal GS, Hoxie SC, Rispoli DM, Steinmann SP. Precontoured parallel plate fixation of AO/OTA type $\mathrm{C}$ distal humerus fractures. J Orthop Trauma. 2009;23(8):575-80.

5. McKee MD, Veillette CJ, Hall JA, et al. A multicenter, prospective, randomized, controlled trial of open reduction: internal fixation versus total elbow arthroplasty for displaced intraarticular distal humeral fractures in elderly patients. J Shoulder Elbow Surg. 2009;18(1):3-12.

6. Worden A, Ilyas AM. Ulnar neuropathy following distal humerus fracture fixation. Orthop Clin North Am. 2012;43(4):50914.

7. Chen RC, Harris DJ, Leduc S, Borrelli JJ Jr, Tornetta P 3rd, Ricci WM. Is ulnar nerve transposition beneficial during open reduction internal fixation of distal humerus fractures? J Orthop Trauma. 2010;24(7):391-4.

8. Wang KC, Shih HN, Hsu KY, Shih $\mathrm{CH}$. Intercondylar fractures of the distal humerus: routine anterior subcutaneous transposition of the ulnar nerve in a posterior operative approach. J Trauma. 1994;36(6):770-3.

9. Vazquez O, Rutgers M, Ring DC, Walsh M, Egol KA. Fate of the ulnar nerve after operative fixation of distal humerus fractures. J Orthop Trauma. 2010;24(7):395-9.

10. Ruan HJ, Liu JJ, Fan CY, Jiang J, Zeng BF. Incidence, management, and prognosis of early ulnar nerve dysfunction in type $\mathrm{C}$ fractures of distal humerus. J Trauma. 2009;67(6):1397-401. 